\title{
Using a discrete event simulation as an effective method applied in the production of recycled material
}

\author{
Knapčíková, L. ${ }^{a,}{ }^{*}$, Behúnová, A. ${ }^{b}$, Behún, M. $^{\mathrm{c}}$ \\ ${ }^{a}$ Technical University of Košice, Faculty of Manufacturing Technologies with a seat in Prešov, Department of Industrial \\ Engineering and Informatics, Prešov, Slovak Republic \\ ${ }^{\mathrm{b}}$ Technical University of Košice, Faculty of Mining, Ecology, Process Control and Geotechnology, Institut of earth sources, \\ Košice, Slovak Republic \\ 'Technical University of Košice, Faculty of Mining, Ecology, Process Control and Geotechnology, Institut of earth sources, \\ Košice, Slovak Republic
}

\begin{abstract}
A B S T R A C T
Technological processes play an essential task in the enterprise's production system. The behaviour and functioning of these systems cannot be predicted with certainty as they belong to a group of probable determinate structures. Generally, if we wanted to know precisely the behaviour of this condition in advance, we would have to be able to describe them mathematically or observe the action of the system on a real object. By applying discrete event simulation software, we realize the development of environmentally friendly products and using the simulation, we gain the certainty that the planned tasks can be implemented in a given time frame, while the simulation of the production process can help to clearly clarify and better understand the processes. To choose the optimal manufacturing ways of cleaning the fabrics component from waste tyres, we used the Witness discrete event simulation software to determine the usability and time occupancy of individual machines in the production of new fabric-based material. We simulated the ultrasonic method of cleaning the fabrics component from waste tyres and the subsequent creation of the test specimen. After the simulation, the obtained data can be used by a selection of type and number of machines and auxiliary equipment, by numbers of tools and fixtures, and by numbers of transport equipment. Obtained results bring the best layout of the workplace, the optimal dose of input materials and resources used in production. We have identified bottlenecks in the machines with long waiting times. The research priority was to reduce bottlenecks and increase the effectiveness of the entire of production line.
\end{abstract}

\author{
ARTICLE INFO \\ Keywords: \\ Green manufacturing; \\ Recycling; \\ Waste tyres; \\ Discrete event simulation; \\ Witness simulation software; \\ Economic impact; \\ Efficiency; \\ Ultrasonic separation \\ *Corresponding author: \\ lucia.knapcikova@tuke.sk \\ (Knapčíková, L.)
}

\section{Article history:}

Received 12 August 2020

Revised 1 December 2020

Accepted 3 December 2020

\section{References}

[1] Lambert, D.M., Stock, J.R., Ellram, L.M. (2000). Logistika, Computer Press, Prague, Czech Republic.

[2] Drastich, A. (2017). Optimization of material flow by simulation methods, Acta Logistica, Vol. 4, No. 4, 23-26, doi: 10.22306/al.v4i4.76.

[3] Fabricators \& Manufacturers Association, International. For Bridgestone's sustainability, zero landfill is where

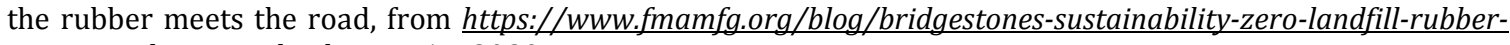
meets-road, accessed February 14, 2020.

[4] Industry 4.0. From http://www.emyras.com/industry-4-0/, accessed July 28, 2020.

[5] Lalinská, J., Kremeňová, I. (2011). Green logistics, In: Proceedings of $12^{\text {th }}$ International Scientific Conference LOGI 2011, Košice, Czech Republic, 252-257, from https://journals.vstecb.cz/wp-content/uploads/2017/02/2011-1.pdf. accessed July 24, 2020. 
[6] Zhang, Y.M., Huang, G.H., He, L. (2011). An inexact reverse logistics model for municipal solid waste management systems, Journal of Environmental Management, Vol. 92, No. 3, 522-530, doi: 10.1016/i.jenvman.2010.09.011.

[7] Sidora, J. (2019). Green logistics, from https://www.ipaslovakia.sk/clanok/green-logistika, accessed November 25, 2019.

[8] Tavakoli, M.M., Haleh, H., Mohammadi, M. (2018). A mathematical model for scheduling of production process and allocation of an automatic guided vehicle in a flexible manufacturing system, International Journal of Engineering Systems Modelling and Simulation, Vol. 10, No. 2, 125-131, doi: 10.1504/IJESMS.2018.091937.

[9] Trebuna, P., Pekarcikova, M., Edl, M. (2019). Digital value stream mapping using the Tecnomatix plant simulation software, International Journal of Simulation Modelling, Vol. 18, No. 1, 19-32, doi: 10.2507/IJSIMM18(1)455.

[10] Rosova, A. (2010). Indices system design of distribution logistics, transport logistics and materials flow as parts of controlling in enterprise's logistics, Acta Montanistica Slovaca, Vol. 15, No. 1, 67-72.

[11] Bai, C., Sarkis, J. (2010). Integrating sustainability into supplier selection with grey system and rough set methodologies, International Journal of Production Economics, Vol. 124, No. 1, 252-264, doi: 10.1016/i.ijpe.2009.11. $\underline{023}$.

[12] Erol, I., Sencer, S., Sari, R. (2011). A new fuzzy multi-criteria framework for measuring sustainability performance of a supply chain, Ecological Economics, Vol. 70, No. 6, 1088-1100, doi: 10.1016/i.ecolecon.2011.01.001.

[13] Crawford, R.J. (1987). Plastics engineering, 2nd edition, Pergamon Press, Oxford, England, doi: 10.1002/zamm. 19890691016.

[14] Harper, C.A. (2000). Modern plastics handbook, 1st edition, McGraw-Hill Professional, New York, USA.

[15] Abdallah, T., Farhat, A., Diabat, A., Kennedy, S. (2011). Green supply chains with carbon trading and environmental sourcing: Formulation and life cycle assessment, Applied Mathematical Modelling, Vol. 36, No. 9, 4271-4285, doi: 10.1016/j.apm.2011.11.056.

[16] Straka, M., Rosová, A., Lenort, R., Besta, P., Šaderová, J. (2018). Principles of computer simulation design for the needs of improvement of the raw materials combined transport system, Acta Montanistica Slovaca, Vol. 23, No. 2, 163-174.

[17] Wang, F., Lai, X., Shi, N. (2011). A multi-objective optimization for green supply chain network design, Decision Support Systems, Vol. 51, No. 2, 262-269, doi: 10.1016/j.dss.2010.11.020.

[18] Straka, M., Khouri, S., Rosova, A., Caganova, D., Culkova, K. (2018). Utilization of computer simulation for waste separation design as a logistics system, International Journal of Simulation Modelling, Vol. 17, No. 4, 583-596, doi: 10.2507/IJSIMM17(4)444.

[19] Vasiliev, V.V., Morozov, E.V. (2001). Mechanics and analysis of composite materials, 1st edition, Elsevier Science, Oxford, United Kingdom.

[20] Knapčíková, L. (2011). Optimizing of technological processes by plastics recovery, Doctoral thesis, Faculty of Manufacturing Technologies of the Technical University of Košice with the seat in Prešov Prešov, Slovakia.

[21] Ivanov, V.A., Feshchenko, A.A. (2019). Simulation of parts manufacturing in the enterprise mechanical-repair department, Journal of Physics: Conference Series, Vol. 1333, No. 9, Article No. 072007, doi: 10.1088/1742-6596/ $1333 / 7 / 072007$.

[22] Okoro, E.E., Iwuajoku, S., Sanni, S.E. (2020). Performance evaluation of biodiesel produced from waste tire pyrolytic oil as a lubricant additive in oil drilling systems, Recycling, Vol. 5, No. 4, Article No. 29, doi: 10.3390/ recycling5040029.

[23] Rupnik, B., Nardin, R., Kramberger, T. (2019). Discrete event simulation of hospital sterilization logistics, Tehnički Vjesnik - Technical Gazette, Vol. 26, No. 5, 1486-1491, doi: 10.17559/TV-20180614102011.

[24] Knapčíková, L., Herzog, M., Oravec, P. (2010). Material characterization of composite materials from used tires, Manufacturing Engineering, Vol. 2010, No. 4, 31-34.

[25] Václavík, V., Daxner, J. (2020). Recovery material use by building insulation (Recyklát slouží při zateplení staveb), from http://www.enviweb.cz/, accessed January 10, 2020.

[26] Taranu, N., Banu, D., Oprisan, G., Budescu, M., Bejan, L. (2013). Strengthening of thin reinforced concrete slabs with composite strips, Romanian Journal of Materials, Vol. 43, No. 1, 3-13.

[27] Schürmann, H. (2001). Konstruieren mit faser-kunststoff-verbunden, Springer, Berlin, Germany.

[28] Dupláková, D., Hatala, M., Duplák, J., Radchenko, S., Steranka, J. (2018). Direct metal laser sintering - Possibility of application in production process, SAR Journal, Vol. 1, No. 4, 123-127, doi: 10.18421/SAR14-01.

[29] Telišková, M., Török, J., Dupláková, D., Kaščák, J., Mezencevová, V., Birčák, J. (2018). Non-destructive diagnostics of hard-to-reach places by spatial digitization, TEM Journal, Vol. 7, No. 3, 612-616, doi: 10.18421/TEM73-18.

[30] Sandin, G., Peters, G.M. (2017). Environmental impact of textile reuse and recycling - A review, Journal of Cleaner Production, Vol. 184, 353-365, doi: 10.1016/j.jclepro.2018.02.266.

[31] Chryssolouris, G. (2006). Manufacturing systems: Theory and practice, 2nd edition, Springer-Verlag, New York, USA.

[32] Müller, M. (2015). Hybrid composite materials on basis of reactoplastic matrix reinforced with textile fibres from process of tyres recyclation, Agronomy Research, Vol. 13, No. 3, 700-708.

[33] Zajac, J., Dupláková, D., Hatala, M., Goldyniak, D., Poklemba, R., Šoltés, P. (2019). The effect of used fillers on the strength characteristics of polymer concrete test bodies, TEM Journal, Vol. 8, No. 3, 795-800, doi: 10.18421/ TEM83-14.

[34] Mohajerani, A., Burnett, L. Smith, J.V., Markovski, S., Rodwell, G., Rahman, M.T., Kurmus, H., Mirzababaei, M., Arulrajah, A., Horpibulsuk, S., Maghool, F. (2020). Recycling waste rubber tyres in construction materials and associated environmental considerations: A review, Resources Conservation and Recycling, Vol. 155, Article No. 104679, doi: 10.1016/j.resconrec.2020.104679. 
[35] Liu, J., Teng, Y., Wang, D., Gong, E. (2019). System dynamic analysis of construction waste recycling industry chain in China, Environmental Science and Pollution Research, Vol. 27, No. 30, 37260-37277, doi: 10.1007/ s11356-019-06739-x.

[36] Kuo, Y.-H., Kusiak, A. (2019). From data to big data in production research: The past and future trends, International Journal of Production Research, Vol. 57, No. 15-16, 4828-4853, doi: 10.1080/00207543.2018.1443230. 


\title{
Uporaba simulacije diskretnih dogodkov kot učinkovite metode $v$ proizvodnji recikliranega materiala
}

\author{
Knapčíková, L. ${ }^{\mathrm{a},}{ }^{,}$, Behúnová, A. ${ }^{\mathrm{b}}$, Behún, M. ${ }^{\mathrm{c}}$ \\ aTechnical University of Košice, Faculty of Manufacturing Technologies with a seat in Prešov, Department of Industrial \\ Engineering and Informatics, Prešov, Slovak Republic \\ ${ }^{\text {b} T e c h n i c a l ~ U n i v e r s i t y ~ o f ~ K o s ̌ i c e, ~ F a c u l t y ~ o f ~ M i n i n g, ~ E c o l o g y, ~ P r o c e s s ~ C o n t r o l ~ a n d ~ G e o t e c h n o l o g y, ~ I n s t i t u t ~ o f ~ e a r t h ~ s o u r c e s, ~}$ \\ Košice, Slovak Republic \\ 'Technical University of Košice, Faculty of Mining, Ecology, Process Control and Geotechnology, Institut of earth sources, \\ Košice, Slovak Republic
}

\section{POVZETEK}

Tehnološki procesi imajo bistveno vlogo $\mathrm{v}$ proizvodnem sistemu podjetja Obnašanja in delovanja proizvodnega sistema podjetja pa ni mogoče $\mathrm{z}$ gotovostjo napovedati, saj gre za strukture, ki so podrejene verjetnosti. Če bi želeli napovedati obnašanje nekega sistema $\mathrm{v}$ realnem okolju, bi morali skrbno opazovati njegovo delovanje in ga matematično natančno opisati. Z uporabo programske opreme za simulacijo diskretnih dogodkov lahko pripomoremo $\mathrm{k}$ boljšemu razumevanju proizvodnega procesa, ugotovimo, ali je načrtovane naloge mogoče izvesti v določenem časovnem okviru ter uresničujemo razvoj okolju prijaznih izdelkov. V tej raziskavi smo za proizvodni proces odstranjevanja tkanine iz odpadnih pnevmatik uporabili programsko opremo za simulacijo diskretnih dogodkov Witness, s pomočjo katere smo ugotovili uporabnost in časovno zasedenost posameznih strojev pri proizvodnji novega materiala na osnovi reciklirane tkanine. Simulirali smo ultrazvočno metodo odstranjevanja tkanine iz odpadnih pnevmatik in kasnejšo izdelavo testnega vzorca. Po simulaciji smo pridobljene podatke uporabili za izbiro vrste in števila strojev, pomožne opreme, kot so orodja in vpenjalne naprave ter števila transportnih naprav in določili najboljšo ureditev delovnega mesta ter optimalno količno vhodnih materialov in virov. Na nekaterih strojih smo prepoznali in zmanjšali ozka grla z dolgimi čakalnimi dobami in povečali učinkovitost celotne proizvodne linije.
\end{abstract}

\section{PODATKI O ČLANKU}

Ključne besede:

Zelena proizvodnja;

Recikliranje;

Odpadne pnevmatike;

Simulacija diskretnih dogodkov;

Programska oprema Witness;

Gospodarski učinek;

Učinkovitost;

Ultrazvočno ločevanje

*Kontaktna oseba: lucia.knapcikova@tuke.sk (Knapčíková, L.)

Zgodovina članka:

Prejet 12. avgusta 2020

Popravljen 1. decembra 2020

Sprejet 3. decembra 2020 